\title{
Timescale-Invariant Representation of Acoustic Communication Signals by a Bursting Neuron
}

\author{
Felix Creutzig, ${ }^{1 \star}$ Sandra Wohlgemuth, ${ }^{1 *}$ Andreas Stumpner, ${ }^{2}$ Jan Benda, ${ }^{3}$ Bernhard Ronacher, ${ }^{1}$ and \\ Andreas V. M. Herz ${ }^{3}$ \\ ${ }^{1}$ Department of Biology and Bernstein Center for Computational Neuroscience Berlin, Humboldt-Universität zu Berlin, 10099 Berlin, Germany, \\ ${ }^{2}$ Georg-August Universität Göttingen, Johann-Friedrich-Blumenbach-Institut für Zoologie und Anthropologie, Abteilung Neurobiologie, 37073 Göttingen, \\ Germany, and ${ }^{3}$ Department of Biology and Bernstein Center for Computational Neuroscience München, Ludwig-Maximilians-Universität München, 82152 \\ Martinsried, Germany
}

Acoustic communication often involves complex sound motifs in which the relative durations of individual elements, but not their absolute durations, convey meaning. Decoding such signals requires an explicit or implicit calculation of the ratios between time intervals. Using grasshopper communication as a model, we demonstrate how this seemingly difficult computation can be solved in real time by a small set of auditory neurons. One of these cells, an ascending interneuron, generates bursts of action potentials in response to the rhythmic syllable-pause structure of grasshopper calls. Our data show that these bursts are preferentially triggered at syllable onset; the number of spikes within the burst is linearly correlated with the duration of the preceding pause. Integrating the number of spikes over a fixed time window therefore leads to a total spike count that reflects the characteristic syllable-to-pause ratio of the species while being invariant to playing back the call faster or slower. Such a timescale-invariant recognition is essential under natural conditions, because grasshoppers do not thermoregulate; the call of a sender sitting in the shade will be slower than that of a grasshopper in the sun. Our results show that timescale-invariant stimulus recognition can be implemented at the single-cell level without directly calculating the ratio between pulse and interpulse durations.

Key words: auditory system; acoustic communication; temporal pattern; spike train; burst code; invariant object recognition

\section{Introduction}

Object recognition relies on the extraction of stimulus attributes that are invariant under natural variations of the sensory input. Such stimulus variations include the size, orientation, or position of a visual object (Sáry et al., 1993; Ito et al., 1995; Rolls, 2000), the strength of an odor (Stopfer et al., 2003), and the intensity, frequency composition, or duration of a sound signal (Shannon et al., 1995; Moore, 1997; Bendor and Wang, 2005; Benda and Hennig, 2008). A particular challenge arises when timescale-invariant features of an acoustic signal are to be extracted because this computation generally involves ratios of temporal quantities. For example, to calculate the relative duration of two sound patterns, the respective durations of both components need to be measured and their ratio calculated.

Many animals use acoustic communication signals to find conspecific mates and judge their reproductive fitness (Gerhardt

Received Oct. 3, 2008; revised Nov. 27, 2008; accepted Dec. 8, 2008.

This work was supported by the German Research Foundation (through Sonderforschungsbereich 618), the Federal Ministry for Education and Research (through the Bernstein Centers for Computational Neuroscience in Berlin and Munich), the Boehringer Ingelheim Fonds, and the German National Merit Foundation. We thank M. Stemmler for helpful comments on this manuscript.

*F.C. and S.W. contributed equally to this work.

This article is freely available online through the J Neurosci Open Choice option.

Correspondence should be addressed to Dr. Andreas V. M. Herz, Department of Biology, Ludwig-MaximiliansUniversität München, Großhaderner Straße 2, 82152 Martinsried, Germany. E-mail: herz@bio.Imu.de. DOI:10.1523/JNEUROSCI.0599-08.2009

Copyright $\odot 2009$ Society for Neuroscience $\quad$ 0270-6474/09/292575-06\$15.00/0 and Huber, 2002). Temporal parameters of songs are speciesspecific and decisive for song recognition in most insects that use acoustic signals (Hennig et al., 2004). For ectothermic species, such as grasshoppers, this poses a severe problem because their calling songs are faster or slower depending on the ambient temperature. Sound signals that are rhythmically structured into syllables and pauses (see Fig. 1A) offer a solution if the receiver uses the temperature-independent syllable-to-pause ratio for species identification, as suggested by behavioral experiments with artificial stimuli (see Fig. $1 \mathrm{~B}$ ). Indeed, the syllable-to-pause ratio varies greatly between different species, facilitating the recognition of conspecific songs (von Helversen and von Helversen, 1994). However, to compute this quantity, durations of syllables and pauses need to be measured and compared with each other. The present study reveals that even a relatively small neural network such as the grasshopper auditory system (Stumpner and Ronacher, 1991; Stumpner et al., 1991) can achieve this task. The underlying computational strategy is surprisingly simple and applicable to a wide range of sensory systems that need to extract timescale-invariant stimulus features in real time.

\section{Materials and Methods}

Experimental paradigms, animals, and electrophysiology. For this study, two different sets of experiments were performed. In the first set, grasshopper mating songs (see Fig. $1 A, C$ ) were presented to six females, three Chorthippus biguttulus, and, for comparison, three Locusta migratoria. Homologous auditory receptor neurons and interneurons have been 
identified in the auditory systems of both species (Römer and Marquart, 1984; Stumpner and Ronacher, 1991). In the second set of experiments, responses to artificial stimuli that mimic the basic syllable-pause structure of the natural songs (see Fig. $1 B$ ) were studied in nine C. biguttulus females. The experimental procedures for both paradigms were similar and are described in detail by Wohlgemuth and Ronacher (2007) and Stumpner and Ronacher (1991), respectively. Briefly, legs, wings, head, and gut were removed, and the animals were fixed with wax, dorsal side up, onto a free-standing holder. The thorax was opened to expose the auditory pathway in the metathoracic ganglion. The whole torso was filled with locust Ringer (Pearson and Robertson, 1981). Neurons were recorded intracellularly; responses were amplified and recorded with a sampling rate of $20 \mathrm{kHz}$. The microelectrode tips were filled with a $3-5 \%$ solution of Lucifer yellow in $0.5 \mathrm{M} \mathrm{LiCl}$. After completion of the stimulation protocols, the dye was injected into the recorded cell by applying hyperpolarizing current. After an experiment, the thoracic ganglia were removed, fixed in $4 \%$ paraformaldehyde, dehydrated, and cleared in methylsalicylate. Stained cells were identified under a fluorescent microscope according to their characteristic morphology. The experimental protocols complied with German laws governing animal care.

Acoustic stimulation. For both experimental paradigms, the stimulusresponse curve of each AN12 cell was determined using 100-ms-long sound pulses whose intensity was varied from 30 to $90 \mathrm{~dB}$ sound pressure level (SPL) in steps of $10 \mathrm{~dB}$. For the core experiments, maximum stimulus intensities were then chosen to lie approximately halfway on the rising slope of the stimulus-response curve. For the natural-song paradigm, songs from eight different males were used: two examples are shown in Figure $1 \mathrm{~A}$. The artificial songs consisted of six syllables with constant sound intensity, separated by silent pauses (see also Fig. $1 B$ ). The durations of syllables $(40,85$, or $110 \mathrm{~ms})$ and pauses $(3.2,8.8,16.3$, $24.0,33.0$, and $42.5 \mathrm{~ms}$ ) were constant for each artificial song and varied in a pseudorandom manner within the experiments. The sound envelopes were filled with a white noise carrier, band passed between 2.5 and $40 \mathrm{kHz}$. Both types of stimuli are known to evoke positive responses in behavioral experiments with female grasshoppers (von Helversen and von Helversen, 1994). Each stimulus set was repeated eight times. Note that stimulus-response curves of AN12 cells have a dynamic range of $\sim 40 \mathrm{~dB}$ (Stumpner and Ronacher, 1991), so that the songs presented (mean SD of the amplitude fluctuations of each song, $10 \mathrm{~dB}$ ) do not extend into the saturated region of the stimulus-response functions.

Data analysis. From the digitized recordings, spike times were determined using a voltage-threshold criterion. To account for neural fatigue, bursts were defined by a recursive formula: a spike belongs to a burst if it follows the preceding, $n$ th, spike of that burst by no more than $(3+n)$ $\mathrm{ms}$. The first interspike interval (ISI) within a burst is thus at most $4 \mathrm{~ms}$, the second at most $5 \mathrm{~ms}$, and so on. This definition reflects the observation that interspike intervals within an AN12 burst typically increase from spike to spike. Accordingly, ISI distributions do not show a clear gap. Using a fixed ISI criterion would therefore either miss the later spikes within a long burst (for short ISI cutoffs) or misclassify isolated spikes as belonging to a multispike burst (for longer ISI cutoffs). Note also that, unlike in other systems (for review, see Krahe and Gabbiani, 2004), the biophysical mechanism underlying AN12 bursting is unknown. For notational simplicity, isolated spikes were treated as bursts with one spike. Throughout this study, we ignored any information in the temporal fine structure within bursts and concentrated on intraburst spike counts (IBSCs). In natural grasshopper songs, each syllable onset is preceded by a period of relative quietness (see Fig. $1 A, C$ ). As for the artificial songs, this period of relative quietness will be called a "pause." It is defined as the interval between the times at which the sound signal, normalized with respect to its mean and SD, passes a certain amplitude threshold from above and again from below. For each cell, the threshold was determined by maximizing the correlation between pause and spike count within the subsequent burst. This procedure leads to a robust threshold estimate (see Fig. 2C). The relative onset amplitude of a specific song syllable is defined as the difference between the maximal amplitude of that syllable and the minimal amplitude in the preceding pause. The total syllable period is calculated as the time interval between the first spikes of two subsequent bursts. Finally, the onset slope is given by the onset amplitude divided by the time interval between maximal and minimal amplitude values. These definitions are illustrated in Figure 1C. To obtain the correlation between spike count and signal features, the $R^{2}$ value (explained variance, Pearson's correlation) was calculated. It was tested for multicollinearity by computing the semipartial $R$ value for each independent variable. The mutual information between pause duration and IBSC was calculated using the adaptive method (Nelken et al., 2005).

Song classification. AN12 responses were classified according to their IBSCs without relying on explicit temporal information: First, the IBSC of each burst was assigned to the time bin $t$ (bin width, $2 \mathrm{~ms}$ ) of the first spike within the burst. Second, for each bin $t$ and song $j$, the mean IBSC value over all repetitions $i$ of the song was calculated. If this mean, $\overline{\operatorname{IBSC}}(j, t)$, exceeded unity, the burst was given a burst position $k(1,2,3, \ldots)$, in ascending order. This procedure was performed for all songs. Third, for every burst position $k$ and repetition $i$, each $\operatorname{IBSC}(i, j, k)$ was assigned to the song $\tilde{j}$ with closest $\overline{\operatorname{IBSC}}(j, k)$, i.e., $\tilde{j}=\arg (\min \hat{j}(\operatorname{diff}(i, j, \hat{j}, k)))$ where $\operatorname{diff}(i, j, \hat{j}, k)=|\operatorname{IBSC}(i, j, k)-\overline{\operatorname{IBSC}}(\hat{j}, k)|$. To avoid a bias, for each $\operatorname{IBSC}(i, j, k)$, the corresponding $\overline{\operatorname{IBSC}}(j, k)$ was calculated without taking $\operatorname{IBSC}(i, j, k)$ into account. Finally, for the cumulative classification, we calculated $\operatorname{diff}_{\text {cum }}\left(i, j, \hat{j}, k^{*}\right)=\sum_{k=1}^{k^{*}} \operatorname{diff}(i, j, \hat{j}, k)$ and assigned each $\operatorname{IBSC}\left(i, j, k^{\star}\right)=\sum_{k=1}^{k^{*}} \operatorname{IBSC}(i, j, k)$ to song $\tilde{j}=\arg \left(\min _{j}\left(\operatorname{diff}_{\text {cum }}\left(i, j, \hat{j}, k^{\star}\right)\right)\right)$. Here, $k^{\star}$ denotes the burst position up to which information is used for cumulative song classification.

Let us emphasize that this analysis of responses to natural grasshopper songs is based on the stationary part of the calling songs, discarding the first nine syllable-and-pause segments of each song. We do so because, during the transient song phase, stimulus amplitudes increase slowly in time. Adaptation processes partially counteract this increase (Machens et al., 2001), leading to approximately equal burst responses on the AN12 level. However, because the duration of transients differs between songs, IBSCs calculated during the initial song phase carry additional information about song identity that reflects the large-scale structure of the song. To focus on the local syllable-pause structure, we analyze only responses to the stationary part of the songs.

\section{Results}

The response properties of a particular neuron (AN12) from the metathoracic ganglion, the first major relay station for auditory signal processing in grasshoppers, may provide an answer to the problem of timescale-invariant sound pattern representation. This neuron belongs to the $\sim 50$ auditory interneurons in the metathoracic ganglion, many of which have been morphologically and physiologically classified (Stumpner and Ronacher, 1994; Stumpner et al., 1991). Ascending neurons (ANs) project into the head ganglion. Some of them (AN1, AN2) encode directional information, whereas others (e.g., AN3, AN4, AN6, AN11, AN12) are presumably involved in pattern recognition (Stumpner and Ronacher, 1994; Krahe et al., 2002). AN12 has a large dynamic range (Stumpner and Ronacher, 1991) of $\sim 40 \mathrm{~dB}$ and generates burst-like discharge patterns when stimulated by the amplitude-modulated sound patterns of grasshopper calling songs (Fig. 1C,D). Its anatomical and stimulus-response characteristics are almost identical in different grasshopper species (Stumpner and Ronacher, 1991; Stumpner et al., 1991; Neuhofer et al., 2008), indicating a highly conserved functional role. For the first part of our study, we recorded from C. biguttulus $(n=3)$ and L. migratoria $(n=3)$, as described in Materials and Methods. Unless stated otherwise, data from C. biguttulus are shown. Under stimulation with natural songs, $67 \%$ of all spikes were contained in bursts with two or more spikes.

Such bursts are mainly generated in response to syllable onsets (Fig. 1D). The IBSC (i.e., number of spikes within a burst) is highly reproducible from trial to trial but varies from syllable to syllable (Fig. 1E). Reflecting the different time courses of different songs (Fig. $1 A$ ), each song thus results in a particular IBSC 
A
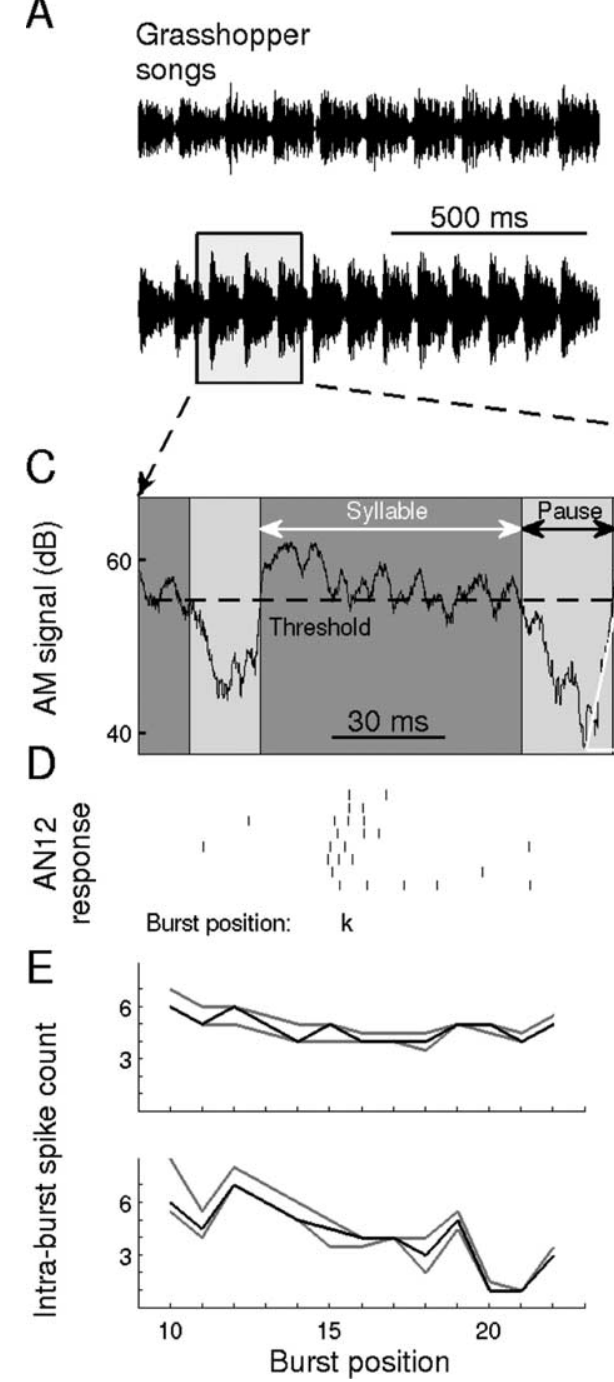

B

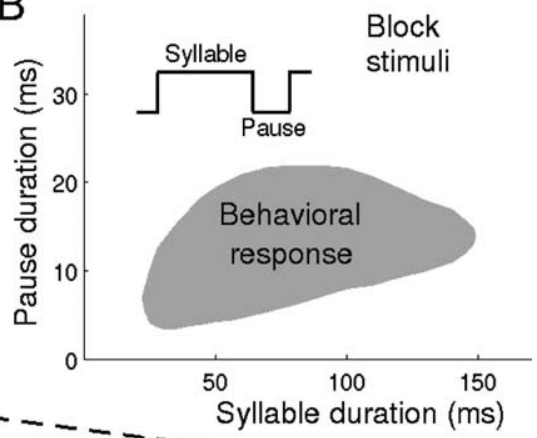

neurons have a far lower overall firing rate and their exact spike timing is neglected within the present analysis. Because IBSCs suffice to discriminate songs even from the same species, this measure must contain useful information about the detailed song structure and thus also help discriminate songs from different species.

Which song features elicit burst activity? To address this question, we constructed burst-triggered stimulus averages (i.e., the mean sound wave preceding a burst with given IBSC). As illustrated in Figure $2 A$, large IBSC values typically occur after extended and deep stimulus excursions, long pauses followed by strong syllable onsets. To identify the song feature that is most important for burst generation, we disentangled the effects of various song parameters (Fig. 1C), such as pause duration, onset amplitude, onset slope, total duration of the preceding song element (syllable plus pause), and minimal pause amplitude. This analysis revealed that pause duration covaries with IBSC (Fig. $2 B)$. The correlation depends on the amplitude level used to define the pause duration, but over a wide range of amplitude levels, it is rather insensitive to parameter variations (Fig. 2C).

To ascertain that pause duration correlates with IBSC under various stimulus conditions, a second set of experiments was performed ( $C$. biguttulus, $n=9$ ). Here, the duration of artificial song units (Fig. $1 B$, inset) was systematically varied (see Materials and Methods) and IBSC increased linearly with pause duration (Fig. 2D). These data also demonstrate that IBSC does not depend on syllable duration. However, IBSC increases with the onset amplitude for natural songs, but with low $R^{2}$ value (Fig. $2 E$, top trace). This effect is attributable to covariations of pause duration and onset amplitude in the grasshopper songs. Finally, IBSC is not systematically influenced by onset slope (Fig. 2E, bottom trace) and all other song features tested. Together, these findings support the hypothesis that the AN12 intraburst spike count is well suited to encode the duration of pauses in grasshopper communication signals.

Could the intraburst spike count be used to reliably transmit information

sequence (Fig. 1E). This signature can be used to discriminate among songs. For example, for a sample with eight songs from one species, each burst carries enough information to assign $\sim 30 \%$ of the responses to the correct song, using the IBSC only (Fig. $1 F$, dashed line). Accumulated over time, a $90 \%$ hit rate is reached after 12 bursts (i.e., $\sim 1 \mathrm{~s}$ ) (Fig. $1 F$, solid line). This astounding discrimination performance is similar to that of grasshopper receptor neurons (Machens et al., 2003), although AN12 about specific structures of the natural calling songs, such as the pause durations, or does the trial-to-trial variability blur the IBSC signal too strongly? To investigate this behaviorally relevant question, we calculated the mutual information between IBSC and preceding pause duration, using the adaptive method (Nelken et al., 2005). On average, the IBSC transmitted $0.49 \pm 0.24$ bits per burst about the pause duration. Hence, a single burst would not convey enough information for a binary classification of pause 
durations. However, grasshoppers can integrate over several subsequent bursts, as shown when they evaluate longer song segments to estimate song quality (von Helversen and von Helversen, 1994). This is useful because trial-to-trial IBSC fluctuations are not significantly correlated from burst to burst in individual cells $[p>0.05$, turning point test (Kendall and Stuart, 1966)]. Consequently, groups of subsequent bursts could be used to encode average pause durations.

Successful auditory discrimination between songs of different species requires the readout of the syllable-to-pause ratio to be independent of the sender's temperature and thus be timescale invariant. Can this task be accomplished with a neural code based on IBSCs? The following consideration helps to answer this question: Any global speed up of a mating song by some factor $X$ increases the number of syllables per second and, hence, the burst frequency by $X$. However, because intraburst spike counts are proportional to the preceding pause durations (Fig. $2 B, D$ ), the IBSC decreases by the same factor $X$. As a result, the total spike count per second stays constant and does not reflect the absolute syllable duration but rather the syllable-to-pause ratio (i.e., the relevant feature for time warp-invariant song pattern recognition) (Fig. $3 A$ ). This prediction is supported by experimental data, as shown by responses from a sample cell to two conspecific grasshopper songs that differ strongly in syllable duration but have almost identical syllable-to-pause ratio (Fig. 3B). Population data from all cells investigated confirm this finding: there is no correlation between the individual timescale of each of the eight songs, given by the duration of one syllable plus one pause, and the elicited total spike count for a given cell (Fig. $3 C$ ). Note that the lower spike count values at $\sim 80 \mathrm{~ms}$ correspond to one specific song. This particular song had a less pronounced syllable-pause structure, which resulted in a lower overall spike count. Integrating the AN12 response over a time window of $\sim 500 \mathrm{~ms}$ or longer thus suffices to reliably estimate the timescale-invariant syllable-to-pause ratio.

\section{Discussion}

Recognition of timescale-invariant stimulus features is a general challenge for sensory systems and seems to imply that ratios are computed in the temporal domain. Here, we showed that this computationally difficult problem can be solved on the singlecell level by a bursting neuron whose output is integrated over a few hundred milliseconds. Temporal integration over such time scales is well known from various neural systems, such as the auditory cortex (Nelken et al., 2003) or the electromotor system of weakly electric fish (Oestreich et al., 2006). The counting all cells tested.
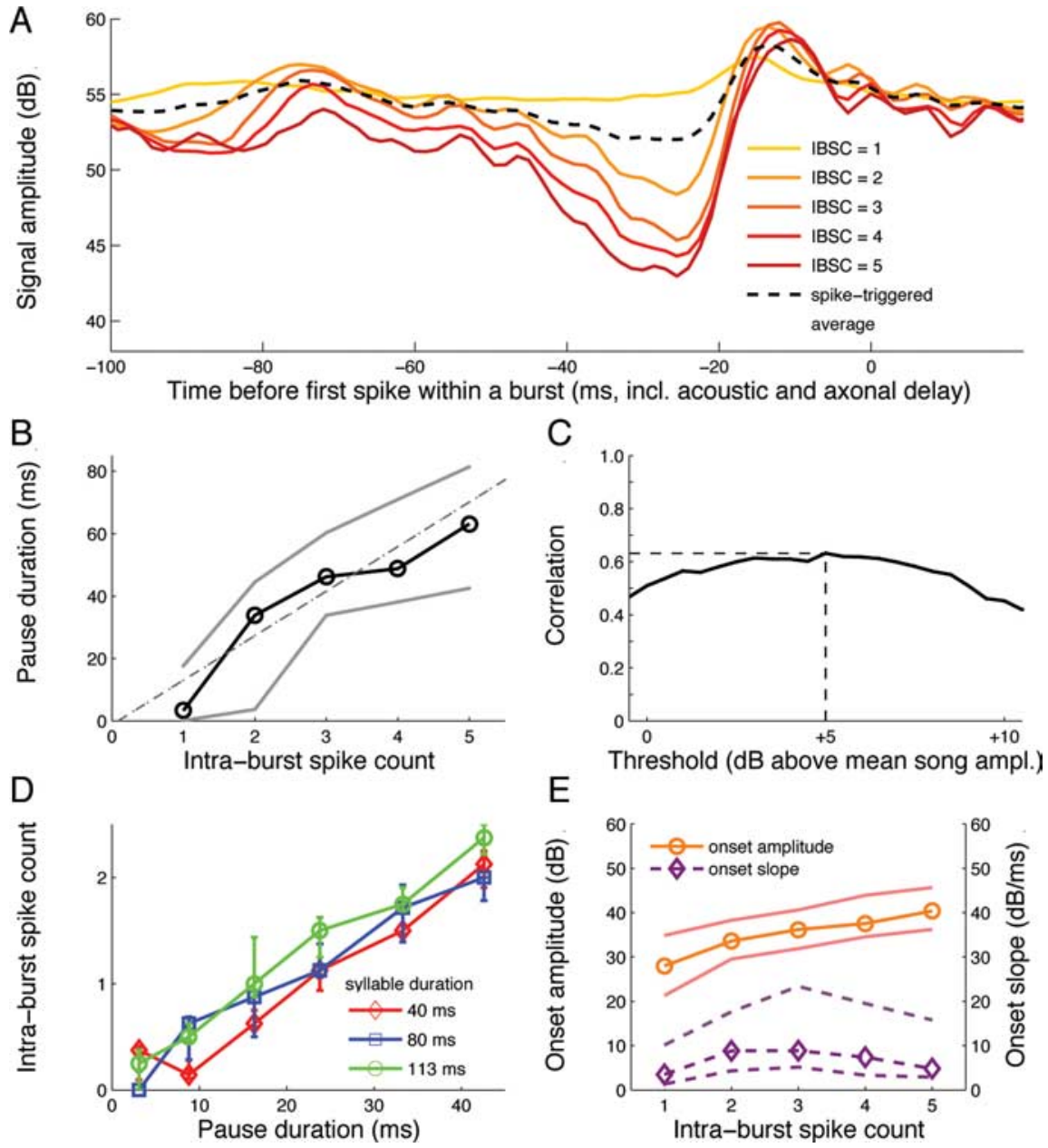

Figure 2. IBSC encodes preceding pause duration. $\boldsymbol{A}$, Burst-triggered average (i.e., mean stimulus before a burst with given IBSC); for comparison, the spike-triggered average over all action potentials is depicted too. As shown by these data, bursts with large IBSC occur preferentially after long phases of low sound intensity. $\boldsymbol{B}$, Pause duration is linearly correlated with the IBSC of the following burst $\left[R^{2}=0.69 \pm 0.15(\mathrm{SD}) ; p<10^{-5}\right.$; Pearson's]; the gray lines indicate upper and lower quartiles. With a $y$-intercept of only $-1.1 \pm 2.1 \mathrm{~ms}$, the best fit agrees with a direct proportionality of pause duration and IBSC. The pause duration was defined individually for each cell by setting a threshold for the sound amplitude level of all songs (Fig. 10); to account for global amplitude differences between the songs, amplitudes were first normalized with respect to mean and SD. C, Correlation coefficient between pause duration and intraburst spike count, as a function of the amplitude threshold used for defining pauses. The smooth maximum demonstrates that the definition in $\boldsymbol{B}$ is robust against variations of the threshold, here shown relative to the mean song amplitude. $\boldsymbol{D}$, For artificial block stimuli, IBSC is again linearly correlated with pause duration $\left(R^{2}=0.76 \pm 0.15 ; p<\right.$ $\left.10^{-5}\right)$. Different colors correspond to stimuli with different syllable durations. $\boldsymbol{E}$, For natural songs, the onset amplitude, but not onset slope, is partially correlated with IBSC $\left(R^{2}=0.27 \pm 0.08 ; p<10^{-5}\right)$ because of covariations of pause duration and onset amplitude in natural songs. The ordinate depicts amplitude (in decibels) for the solid curve, and amplitude/time (in decibels/ millisecond) for the dashed curve. Data in $\boldsymbol{A}-\boldsymbol{C}$ and $\boldsymbol{E}$ are from one sample cell; $\boldsymbol{D}$ refers to a second neuron. The statistics refer to

mechanism proposed for readout is similar to that of frogs that also communicate with rhythmic patterns of sound pulses and quiet intervals. Specific neurons in the frog's auditory midbrain integrate the number of acoustic pulses and respond only if a minimal number of appropriate pulses and interpulse intervals arrive (Alder and Rose, 1998; Edwards et al., 2002, 2007). Combined excitation and inhibition determine the dependence of the responses on the duration of the acoustic elements (Edwards et al., 2008; Leary et al., 2008). Together with these results, our findings suggest that the recognition of temporally extended acoustic objects is supported by neural integration over multiple timescales. This hierarchical integration is reminiscent of current models for invariant object recognition in vision that suggest that high-level invariances are detected by successive spatial integra- 
A

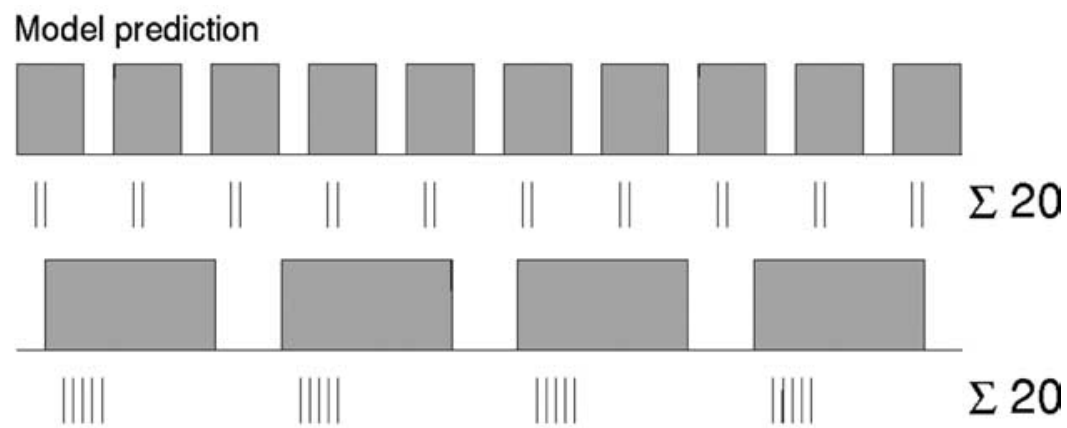

\section{B Experimental data}
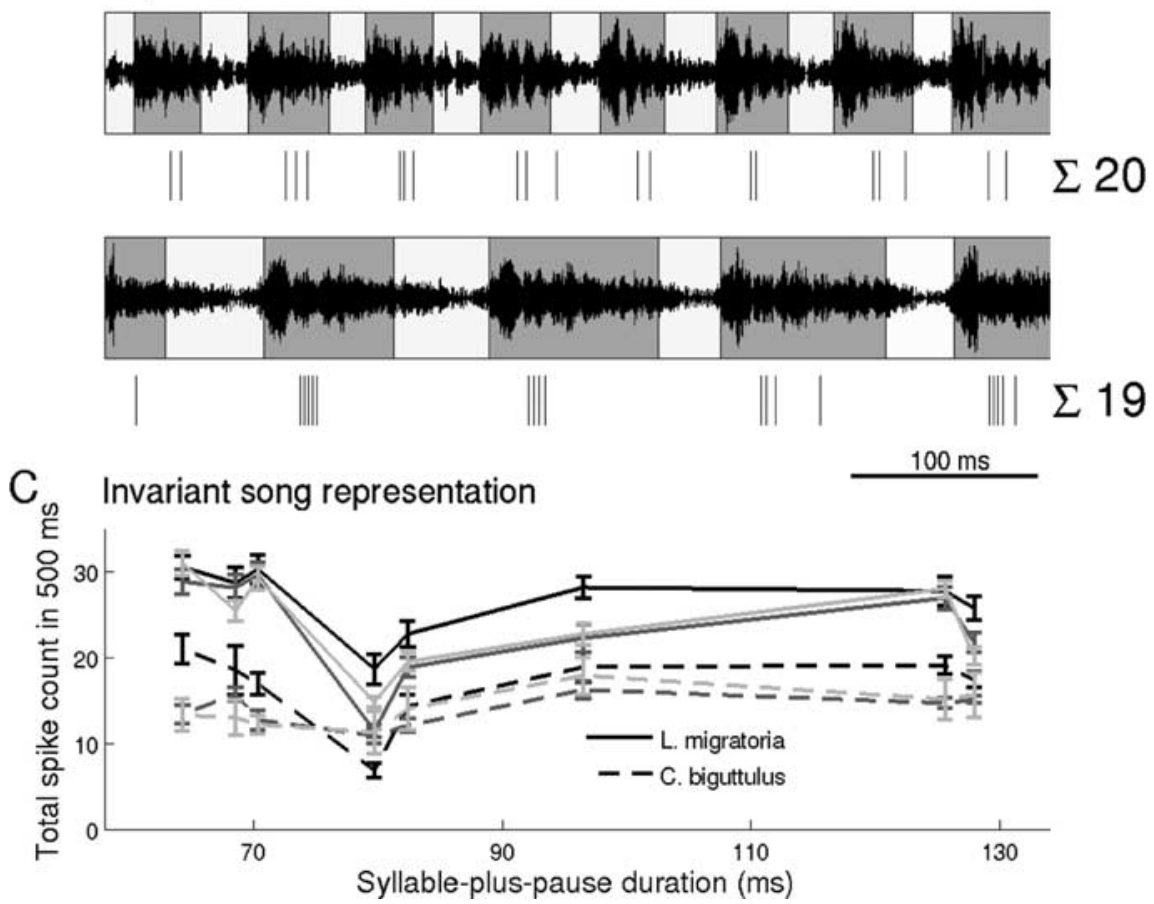

Figure 3. Total spike count is invariant to temporal stimulus rescaling. A, Sketch of two simple auditory sound signals. The lower stimulus is obtained by stretching the upper stimulus by a factor of 2.5. Assuming that the IBSC scales linearly with the preceding pause duration, this rescaling results in 4 instead of 10 bursts, but each burst has now 5 instead of only 2 spikes. Both effects compensate each other so that the total spike count stays constant. $\boldsymbol{B}$, Measured AN12 responses to two conspecific grasshopper songs. Although the songs differ strongly in their pause durations, the total spike count is approximately equal because the syllable-to-pause ratios of both songs are approximately the same (1.41 for the upper and 1.40 for lower song, respectively), in agreement with the model prediction. C, Total spike count (window length, $500 \mathrm{~ms}$ ) of responses to the eight tested songs, as a function of the duration of one syllable plus one pause. The duration of this basic song element differs for each song, resulting in the eight different values on the abscissa. Each of the six curves represents data from one neuron, with the species indicated by line type. Error bars indicate upper and lower quartiles of total spike count across eight repetitions. The total spike count varies from cell to cell but without overall correlation to the syllable-plus-pause duration $\left(R^{2}=0.00 \pm 0.14\right)$. Downstream neurons thus have access to the behaviorally relevant syllable-to-pause ratio in a timescale-invariant manner. relevance of a particular sound pattern. Using pause duration instead of syllable duration may be beneficial because this strategy lowers the overall dependency on sound intensity. Although the timescaleinvariant readout mechanism proposed by this study depends in principle on the existence of "pauses" in an atonal noise signal, this mechanism can be easily extended to stimuli with rich tonal structure if the raw signal is first split into multiple frequency bands. For rhythmic stimuli in acoustic environments with background noise, separately analyzing specific frequency components using the mechanism proposed in this study will help improve the signal-to-noise ratio in frequency bands with low noise content. Likewise, in mixtures of stimuli with different rhythms and carrier frequencies, a frequencyresolved analysis may reveal otherwise hidden signal components. Computationally demanding divisions in the time domain are thus not required to extract timescale-invariant information from sensory stimuli.

\section{References}

Alder TB, Rose GJ (1998) Long-term temporal integration in the anuran auditory system. Nat Neurosci 1:519-523.

Arganda S, Guantes R, de Polavieja GG (2007) Sodium pumps adapt spike bursting to stimulus statistics. Nat Neurosci 10:1467-1473.

Benda J, Hennig RM (2008) Spike-frequency adaptation generates intensity invariance in a primary auditory interneuron. J Comput Neurosci 24:113-136.

Bendor D, Wang X (2005) The neuronal representation of pitch in primate auditory cortex. Nature 436:1161-1165.

Edwards CJ, Alder TB, Rose GJ (2002) Auditory midbrain neurons that count. Nat Neurosci 5:934-936.

Edwards CJ, Leary CJ, Rose GJ (2007) Counting on inhibition and rate-dependent excitation in the auditory system. J Neurosci 27:13384-13392.

Edwards CJ, Leary CJ, Rose GJ (2008) Mechanisms of long-interval selectivity in midbrain auditory neurons: roles of excitation, inhibition and plasticity. J Neurophysiol 100:3407-3416.

Gerhardt HC, Huber F (2002) Acoustic commution over lower-level stimulus features (Riesenhuber and Poggio, 1999).

It has been suggested that bursts can represent signal slope (Kepecs et al., 2002). In fact, IBSC codes the relative slope of skin displacement in leech (Arganda et al., 2007) and the integral of rate of change in peak pressure in the auditory cortex (Heil, 1997). In contrast to these findings, our data show that the IBSCs of the AN12 neuron do not encode the slope of a stimulus transient but rather the duration of a quiescent stimulus segment. In addition, this time interval is recoded in that a pause of $40 \mathrm{~ms}$ may be represented by a burst that lasts for 4 ms only. The temporal compression makes it possible that this new burst code can multiplex information about the time of occurrence and behavioral nication in insects and anurans. Chicago: University of Chicago.

Heil P (1997) Auditory cortical onset responses revisited. II. Response strength. J Neurophysiol 77:2642-2660.

Hennig RM, Franz A, Stumpner A (2004) Processing of auditory information in insects. Microsc Res Tech 63:351-374.

Ito M, Tamura H, Fujita I, Tanaka K (1995) Size and position invariance of neuronal responses in monkey inferotemporal cortex. J Neurophysiol 73:218-226.

Kendall MG, Stuart A (1966) The advanced theory of statistics, Vol III. London: Griffin.

Kepecs A, Wang XJ, Lisman J (2002) Bursting neurons signal input slope. J Neurosci 22:9053-9062.

Krahe R, Gabbiani F (2004) Burst firing in sensory systems. Nat Rev Neurosci 5:13-23. 
Krahe R, Budinger E, Ronacher B (2002) Coding of a sexually dimorphic song feature by auditory interneurons of grasshoppers: the role of leading inhibition. J Comp Physiol A Neuroethol Sens Neural Behav Physiol 187:977-985.

Leary CJ, Edwards CJ, Rose GJ (2008) Midbrain auditory neurons integrate excitation and inhibition to generate duration selectivity: an in vivo whole-cell patch study in anurans. J Neurosci 28:5481-5493.

Machens CK, Stemmler MB, Prinz P, Krahe R, Ronacher B, Herz AVM (2001) Representation of acoustic communication signals by insect auditory receptor neurons. J Neurosci 21:3215-3227.

Machens CK, Schütze H, Franz A, Kolesnikova O, Stemmler MB, Ronacher B, Herz AV (2003) Single auditory neurons rapidly discriminate conspecific communication signals. Nat Neurosci 6:341-342.

Moore BCJ (1997) An introduction to the psychology of hearing, Ed 4. New York: Academic.

Nelken I, Fishbach A, Las L, Ulanovsky N, Farkas D (2003) Primary auditory cortex of cats: feature detection or something else? Biol Cybern 89:397-406.

Nelken I, Chechik G, Mrsic-Flogel TD, King AJ, Schnupp JWH (2005) Encoding stimulus information by spike numbers and mean response time in primary auditory cortex. J Comput Neurosci 19:199-221.

Neuhofer D, Wohlgemuth S, Stumpner A, Ronacher B (2008) Evolutionarily conserved coding properties of auditory neurons across grasshopper species. Proc R Soc Biol Sci 275:1965-1974.

Oestreich J, Dembrow NC, George AA, Zakon HH (2006) A "sample-andhold" pulse-counting integrator as a mechanism for graded memory underlying sensorimotor adaptation. Neuron 49:577-588.

Pearson KG, Robertson RM (1981) Interneurons coactivating hindleg flexor and extensor motoneurons in the locust. J Comp Physiol 144:391-400.
Riesenhuber M, Poggio T (1999) Hierarchical models of object recognition in cortex. Nat Neurosci 2:1019-1025.

Rolls ET (2000) Functions of the primate temporal lobe cortical visual areas in invariant visual object and face recognition. Neuron 27:205-218.

Römer H, Marquart V (1984) Morphology and physiology of auditory interneurons in the metathoracic ganglion of the locust. J Comp Physiol 155:249-262.

Sáry G, Vogels R, Orban GA (1993) Cue-invariant shape selectivity of macaque inferior temporal neurons. Science 260:995-997.

Shannon RV, Zeng FG, Kamath V, Wygonski J, Ekelid M (1995) Speech recognition with primarily temporal cues. Science 270:303-304.

Stopfer M, Jayaraman V, Laurent G (2003) Intensity versus identity coding in an olfactory system. Neuron 39:991-1004.

Stumpner A, Ronacher B (1991) Auditory interneurones in the metathoracic ganglion of the grasshopper Chorthippus biguttulus. I. Morphological and physiological characterization. J Exp Biol 158:391-410.

Stumpner A, Ronacher B (1994) Neurophysiological aspects of song pattern recognition and sound localization in grasshoppers. Am Zool 34:696-705.

Stumpner A, Ronacher B, von Helversen O (1991) Auditory interneurones in the metathoracic ganglion of the grasshopper Chorthippus biguttulus. II. Processing of temporal patterns of the song of the male. J Exp Biol 158:411-430.

von Helversen O, von Helversen D (1994) Forces driving coevolution of song and song recognition in grasshoppers. Fortschr Zool 39:253-284.

Wohlgemuth S (2008) Representation and discrimination of amplitudemodulated acoustic signals in the grasshopper nervous system. Dissertation, Humboldt-Universität zu Berlin.

Wohlgemuth S, Ronacher B (2007) Auditory discrimination of amplitude modulations based on metric distances of spike trains. J Neurophysiol 97:3082-3092. 